\title{
Trans-scale characterization of interface fracture in peel test for metal film/ceramic substrate systems
}

\author{
Jingru Song ${ }^{\mathrm{a}}$, Yueguang $\mathrm{Wei}^{\mathrm{b}, *}$ \\ ${ }^{a}$ State-Key Laboratory of Nonlinear Mechanics, Institute of Mechanics, Chinese Academy of Sciences, Beijing 100190, PR China \\ ${ }^{\mathrm{b}}$ Department of Mechanics, College of Engineering, Peking University, Beijing 100871, PR China
}

\section{A R T I C L E I N F O}

\section{Keywords:}

Interfacial separation strength

Trans-scale mechanics theory

Size effect

Peel test

Length scale parameter

\begin{abstract}
A B S T R A C T
In order to describe the interfacial fracture behaviors of the metal thin film with nano- or microscale thickness peeled on the ceramic substrate, a trans-scale mechanics model has been adopted. In the trans-scale mechanics model, both the strain gradient effect and surface/interface effect are considered. In addition, two fracture process models are used in present study, which are the cohesive zone model and the virtual internal bond model. Using the trans-scale mechanics theory and the interface models, the size effect of the interfacial separation strength between the metal thin films and the ceramic substrates is analyzed systematically by using the peel test. The results show that the fracture process zone size could be taken as the indicator of the trans-scale interface fracture characterization. The interface effect should be considered when the fracture process zone size is at the nanoscale, and the obtained interfacial separation strength is much higher than the conventional separation strength. The material length scale parameters of the metal films are determined by comparing the interfacial energy release rate predicted by the scale theories with the experimental results, which shows that the material length scale parameter could be regarded as the size of active plastic zone in the small scale yielding case during the peeling process.
\end{abstract}

\section{Introduction}

In last several decades, the thin film/substrate structures and materials have undergone a wide applications in micro/nanoengineering, such as microelectronic device designing [1,2], thermal barrier coating protection [3,4], and modern material manufacturing $[5,6]$, etc. The interface adhesive debonding, stripping and delamination etc. are the main failure behaviors of these structures, and the interface fracture properties are dominated by the interfacial strength and the interfacial fracture toughness.

Researches on the interface fracture behaviors between thin films and substrates have been attracting a great deal of attention [7-16]. It has been found that some peculiar failure phenomena exhibited by crack propagation along the interface cannot be explained well by the conventional elastoplastic theory. For example, the interfacial separation strength very near to the crack tip of the metal/ceramic interface observed by the experiment can reach several times or even ten times the yield strength of the material at macroscopic scale, but the corresponding value predicted by the conventional elastoplastic theory can only reach three or four times at most [13-15]. As the film thickness decreases, the size of the singular stress dominant zone is also reduced accordingly [16]. For the case of micro/nano-scale film thickness, stress singular regions are generally only tens to hundreds of nanometers in size. In this case, it is also debatable whether the concept of classical fracture mechanics can be applied.

\footnotetext{
* Corresponding author.

E-mail address: weiyg@pku.edu.cn (Y. Wei).
} 
Peel test is one of the most important methods to measure the interface fracture properties, mainly for analyzing the interfacial separation strength and the interfacial energy release rate between thin films and substrates. There have been a lot of work on the peel test in experiments, modeling and simulations [17-25]. For an elastic thin film on a rigid substrate, Kendall [17] developed a peeling model for the steady-state peeling case, and was later extended to the whole peeling stage and to the viscoelastic film case [18-20]. To analyze the peeling behavior of elastic-plastic thin films, Kim and his coworkers [21] presented a plastic bending model to predict the plastic dissipation of ductile thin films, following that with most analyses on ductile thin film peeling adopted or referred to this bending model. In order to model the peeling behavior of films more comprehensively, Wei et al. [22-24] used the elastoplastic large deformation beam bending model combined with the rigorous plane-strain finite element analysis to characterize the ductile film peeling, and the interface between the film and the substrate was described by using the cohesive zone model. The results they obtained are quite different from the Kim model. Later, some other factors, such as interfacial wavy morphologies, were also considered through the cohesive zone model [25]. It shows that the cohesive stress has a great influence on the fracture process and the plastic deformation of the material near the crack tip. Therefore, the cohesive stress should be considered in the prediction of interfacial fracture toughness. But even so, the above combined calculation method will become invalid for the interface with very high separation strength (about more than 4-6 times yield strength). The reason is that the traditional elastoplastic $\mathrm{J}_{2}$ flow theory underestimates the stress at the crack tip, causing the crack propagation delay or not to occur at all [22]. In this case, strain gradient plasticity theory is needed to describe the constitutive relationship of the thin film, especially for microscale or even nanoscale peeling process [26].

Adopting the framework of conventional plasticity theory, the strain gradient plastic theory [27-29] considers the strain gradient effect, which can better describe the size effect in the peeling process. The strain gradient terms appearing in the constitutive equations of the strain gradient theory match with the conventional strain terms by introducing a kind of length parameters which are called material length scales. Analyses for thin wire torsion and micro bending etc. showed that the significant influence of the strain gradient effect was under microscale of specimen size and the length parameters included in the theory were on the order of micrometers $(0.1-10 \mu \mathrm{m})$ [30,31]. Wei and Hutchinson [27] applied the strain gradient plasticity to analyze the mode I steady-state crack growth. The results showed that the separation stress ahead of the crack tip can attain significantly higher levels than predicted by conventional plasticity, about ten times more than the yield stress. Then, by adopting the theory, the nonlinear degumming problem of metal films along ceramic substrates was analyzed, and the material length scale parameters were also be estimated [32].

The above models and theories more reasonably describe the interface fracture behaviors in the peel test at micro-scales to a certain extent, but they all ignore the influence of the surface/interface effects. However, when the fracture process zones are at nanometer scale, the surface/interface effects are important and cannot be neglected compared to the strain gradient effect. Later, Wei et al. $[33,34]$ proposed a trans-scale mechanics (TSM) theory, in which both strain gradient effect and surface/interface effect are considered, so that it can be used to characterize the trans-scale mechanical behaviors for the micro-/nanostructured materials. Within the framework of continuum mechanics, Yao et al. $[35,36]$ developed an elasticity theory for nanomaterials using the concept of the surface/interface energy density. In the present paper, we intend to systematically analyze the trans-scale behaviors of the interface fracture in peel test for the metal thin film/ceramic substrate systems, by combining the trans-scale mechanics theory and two common models of simulating interface fracture, namely the cohesive zone model and the virtual internal bond model. The influence zone of interfacial effect will be implemented. In addition, the values of the material length scale parameters of the metal materials will also be determined by comparing the experimental results of the peel test of thin metal film along ceramic substrate with the prediction results based on the trans-scale mechanics theory.

\section{Trans-scale mechanics (TSM) theory}

Considering both strain gradient effect and surface/interface effect, the variation equation of the total potential for any deformation solid system can be expressed as follows [33,34]

$$
\int_{V}(\sigma: \delta \varepsilon+\tau \vdots \delta \eta) d V+\delta \int_{S^{\prime}} \gamma d S^{\prime}-\int_{V} \overline{\boldsymbol{f}} \cdot \delta \boldsymbol{u} d V-\int_{S}\left[\overline{\boldsymbol{t}} \cdot \delta \boldsymbol{u}+\overline{\boldsymbol{S}}^{s}: \delta \boldsymbol{\varepsilon}^{s}+\overline{\boldsymbol{r}} \cdot(\delta \boldsymbol{u n} \cdot \nabla)\right] d S=0
$$

where $(\sigma, \varepsilon, \boldsymbol{u}, \overline{\boldsymbol{t}}, \overline{\boldsymbol{r}}, \overline{\boldsymbol{f}})$ are the tensor or vector of stress, strain, displacement, traction, torque and volume force; $\tau$ and $\eta$ are the tensors of higher-order stress and strain gradient. For the interface problem, $\gamma$ denotes the interface energy density; $S^{\prime}$ is interface area of the current configuration after deformation; $\delta \int_{S^{\prime}} \gamma d S^{\prime}$ is the variation of interface energy calculated along the transient interface area; $\overline{\boldsymbol{S}}^{s}$ and $\boldsymbol{\varepsilon}^{s}$ denotes the interface stress and strain along the interface direction.

\subsection{Fundamental relations of Trans-scale theory only considering interface effect}

When the interface effect is considered only, from Eq. (1), the TSM theory can be described as follows

$$
\int_{V} \sigma: \delta \varepsilon d V+\delta \int_{S^{\prime}} \gamma d S^{\prime}-\int_{V} \overline{\boldsymbol{f}} \cdot \delta \boldsymbol{u} d V-\int_{S}\left[\overline{\boldsymbol{t}} \cdot \delta \boldsymbol{u}+\overline{\boldsymbol{S}}^{s}: \delta \varepsilon^{s}\right] d S=0
$$

In order to describe the interface effect clearly, we need to define the interface strain for the peel test problem first. As shown in Fig. 1, the cohesive zone model is used to describe the fracture process zone, the basis vectors along the interface inside normal direction and the interface direction are $e_{m}$ and $e_{l}$, respectively. The displacement can be expressed as $\boldsymbol{u}=u_{s}(l, m) \boldsymbol{e}_{l}+u_{m}(l, m) \boldsymbol{e}_{m}$, and the tensor of the strain is $\varepsilon=\frac{1}{2}\left[\nabla u+(\nabla u)^{\mathrm{T}}\right]$, where $\nabla=\boldsymbol{e}_{l} \frac{\partial}{\partial l}+\boldsymbol{e}_{m} \frac{\partial}{\partial m}$. We can therefore get the interface strain, 


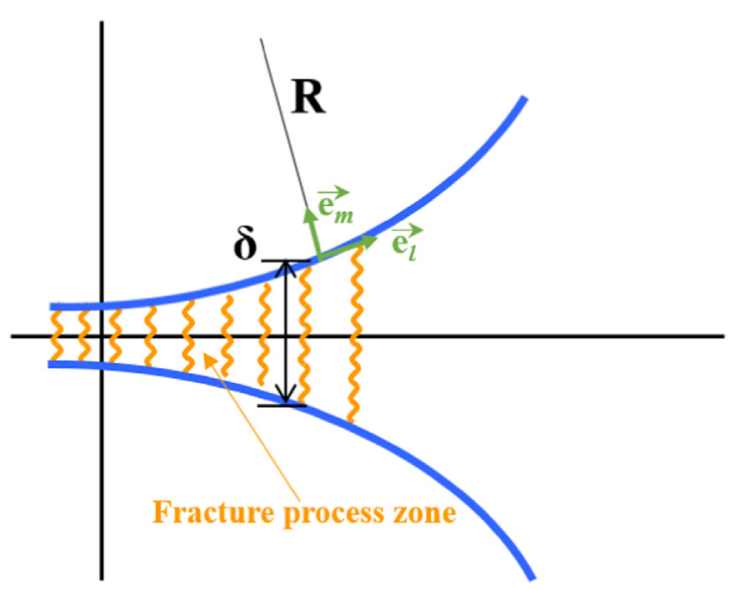

Fig. 1. The diagram of interface deformation and fracture.

$$
\boldsymbol{\varepsilon}^{s}=\left(u_{s, l}^{\prime}-\frac{u_{m}}{R}\right) \boldsymbol{e}_{l} \boldsymbol{e}_{l}
$$

where $R$ is the curvature radius after interface fracture.

For describing the finite deformation of thin film, the Jacobian determinant of deformation along the interface direction is introduced and expressed as $J^{s}$, we have $J^{s}=1+\varepsilon^{s}: \boldsymbol{I}^{s}$, where $\boldsymbol{I}^{s}=\boldsymbol{e}_{l} \boldsymbol{e}_{l}$ is the unit tensor along the interface direction. The Taylor expansion of the interface energy density at the interface strain $\varepsilon^{s}=\mathbf{0}$ is

$$
\gamma=\gamma_{0}+\boldsymbol{f}_{0}^{s}: \boldsymbol{\varepsilon}^{s}+\frac{1}{2} \varepsilon^{s}: \boldsymbol{E}^{s}: \boldsymbol{\varepsilon}^{s}
$$

where $\gamma_{0}$ is a constant, which is the interface energy density value independent of the interface strain; $\boldsymbol{f}_{0}^{s}$ and $\boldsymbol{E}^{s}$ are the surface stresses corresponding to the primary and secondary terms of the interface strain, respectively. $\boldsymbol{f}^{\boldsymbol{s}}$ is the derivative of interface energy density to interface strain, given by

$$
\boldsymbol{f}^{s}=\frac{\partial \gamma}{\partial \varepsilon^{s}}=\boldsymbol{f}_{0}^{s}+\boldsymbol{E}^{s}: \boldsymbol{\varepsilon}^{s}
$$

Therefore, the interface energy of different configurations before and after interface fracture have the following relationship,

$$
\delta \int_{S^{\prime}} \gamma d S^{\prime}=\int_{S} \delta\left(\gamma J^{s}\right) d S=\int_{S}\left[J^{s} \frac{\partial \gamma}{\partial \varepsilon^{s}} \delta \varepsilon^{s}+\gamma \frac{\partial J^{s}}{\partial \varepsilon^{s}} \delta \varepsilon^{s}\right] d S=\int_{S}\left[J^{s} \boldsymbol{f}^{s}+\gamma \boldsymbol{I}^{s}\right] \delta \varepsilon^{s} d S
$$

Considering the interface constitutive relationship, we have

$$
\delta \int_{S^{\prime}} \gamma d S^{\prime}=\int_{S} S^{s} \delta \varepsilon^{s} d S
$$

Comparing Eq. (6) and Eq. (7), the interface stress can be expressed as

$$
\boldsymbol{S}^{s}=J^{s} \boldsymbol{f}^{s}+\gamma \boldsymbol{I}^{s}
$$

Substituting Eq. (3) into Eq. (5) and (8), we have

$$
\begin{gathered}
\boldsymbol{f}^{s}=\left[\boldsymbol{f}_{0}^{s}+E_{s}\left(u_{s, l}^{\prime}-\frac{u_{m}}{R}\right)\right] \boldsymbol{e}_{l} \boldsymbol{e}_{l} \\
\boldsymbol{S}^{s}=J^{s} \boldsymbol{f}^{s}+\gamma \boldsymbol{I}^{s}=\left\{\left(1+u_{s, l}^{\prime}-\frac{u_{m}}{R}\right)\left[\boldsymbol{f}_{0}^{s}+E_{s}\left(u_{s, l}^{\prime}-\frac{u_{m}}{R}\right)\right]+\gamma\right\} \boldsymbol{e}_{l} \boldsymbol{e}_{l}
\end{gathered}
$$

So, the interface work variation is

$$
\boldsymbol{S}^{s}: \delta \varepsilon^{s}=\left\{\left(1+u_{s, l}^{\prime}-\frac{u_{m}}{R}\right)\left[\boldsymbol{f}_{0}^{s}+E_{s}\left(u_{s, l}^{\prime}-\frac{u_{m}}{R}\right)\right]+\gamma\right\}\left(\delta u_{s, l}^{\prime}-\frac{\delta u_{m}}{R}\right)
$$

Considering the balance along $m$ direction, the interfacial separation stress is

$$
t_{m}=t_{m}^{-}+\left\{\left(1+u_{s, l}^{\prime}-\frac{u_{m}}{R}\right)\left[f_{0}^{s}+E_{s}\left(u_{s, l}^{\prime}-\frac{u_{m}}{R}\right)\right]+\gamma\right\} \frac{1}{R}
$$

where $\bar{t}_{m}$ is the conventional interfacial cohesive stress, and can be calculated by the cohesive zone model and the virtual internal bond model, as seen in Appendix A. It can be seen that when the fracture process zone is relatively small (at nanoscale), the curvature of the crack interface $1 / R$ becomes very large, therefore, the influence of the interface effect needs to be considered. The fracture 
process zone at the nanometer scale can be described by the TSM theory. If the interface constitutive relationship is not considered, Eq. (11) can be simplified as

$$
t_{m} \approx \bar{t}_{m}+\gamma / R
$$

Substituting Eq. (A.7) in Appendix A into the above equation, we can get the interfacial separation stress considering the interface effect as follows

$$
t_{m} \approx \bar{t}_{m}+\gamma / R=\sigma+\gamma / R=\left(\frac{\hat{\sigma}^{2} \cdot e^{2}}{\Gamma}\right) \cdot \delta \cdot \exp \left(-\frac{\delta \cdot \hat{\sigma} \cdot e}{\Gamma}\right)+\frac{\gamma}{R}
$$

where the curvature of the crack interface $1 / R$ can be calculated by

$$
1 / R=\left|\frac{d^{2} u_{m}}{d x^{2}} /\left[1+\left(\frac{d u_{m}}{d x}\right)^{2}\right]^{3 / 2}\right|
$$

where $u_{m}=(1 / 2) \cdot \delta$ is the displacement along $m$ direction.

\subsection{Fundamental relations of trans-scale theory only considering strain gradient effect}

When the strain gradient effect is considered only, from Eq. (1), the TSM theory can be described as follows

$$
\int_{V}(\sigma: \delta \varepsilon+\tau \vdots \delta \eta) d V-\int_{V} \overline{\boldsymbol{f}} \cdot \delta \boldsymbol{u} d V-\int_{S}[\overline{\boldsymbol{t}} \cdot \delta \boldsymbol{u}+\overline{\boldsymbol{r}} \cdot(\delta \boldsymbol{u n} \cdot \nabla)] d S=0
$$

It is the strain gradient plasticity theory. The general compressible deformational theory of Fleck-Hutchinson strain gradient version [28] has been presented by Wei et al. $[27,29]$. The constitutive relations can be expressed as follows

$$
\begin{aligned}
& \sigma_{i j}=\frac{E}{1+\nu+\frac{3}{2} E / h^{p}} \varepsilon_{i j}+\frac{1}{3}\left(\frac{E}{1-2 \nu}-\frac{E}{1+\nu+\frac{3}{2} E / h^{p}}\right) \varepsilon_{k k} \delta_{i j}, \\
& \tau_{i j k}=2 E\left\{\sum_{I=1}^{3} \frac{l_{I}^{2}}{l_{I}^{2} / l_{I}^{22}+2 E / h^{p}} T_{i j k l m n}^{(I)}+l_{4}^{e 2} T_{i j k l m n}^{(4)}\right\} \eta_{l m n},
\end{aligned}
$$

where $E$ and $\nu$ are the Young's modulus and Poisson's ratio, respectively, $l_{I}^{e}(I=1,4)$ and $l_{I}(I=1,3)$ are the material length scale parameters of strain gradient elasticity and plasticity, respectively.

The strain $\varepsilon_{i j}$ and the strain gradient $\eta_{i j k}$ are defined by

$$
\varepsilon_{i j}=\frac{1}{2}\left(u_{i, j}+u_{j, i}\right), \eta_{i j k}=u_{k, i j}
$$

The elastic parts of them are conjugated with the stress $\sigma_{i j}$ and the higher-order stress $\tau_{i j k}$ through the elastic strain energy density, respectively. The plastic parts of them can be expressed as

$$
\varepsilon_{i j}^{p}=\frac{3}{2 h^{p}} \sigma_{i j}^{\prime}, \eta_{i j k}^{p}=\frac{1}{h^{p}} \sum_{I=1}^{3} l_{I}^{-2} T_{i j k l m n}^{(I)} \tau_{l m n},
$$

where $h^{p}$ is the plastic modulus, $T_{i j k l m n}^{(I)}(I=1,4)$ is the projection tensor of the strain gradients, and the detailed expressions have been presented by Ref. [27]. For more general solid, there is an approximate relation among the material length scale parameters [28]

$$
l_{1}=0, l_{2}=\frac{1}{2} l, l_{3}=\sqrt{\frac{5}{24} l} .
$$

In the present work, for simplified analysis, the material length scale parameters are taken as the same value $l$.

The finite element method for Wei-Hutchinson strain gradient theories was presented by Ref. [29], and has been successfully used to analyze the fundamental fracture problems. Through this numerical method, the interfacial energy release rate of metal thin film delamination along ceramic substrate was obtained, which will be used to analyze the results of the peel test in our present work (as seen in Section 4).

\section{Interfacial separation strength of peel test considering interface effect}

Considering a systematical peel test, $\mathrm{Al}$ and $\mathrm{Cu}$ films with a series of thicknesses between 20 and $250 \mu \mathrm{m}$ bonded to a ceramic substrate $\left(\mathrm{Al}_{2} \mathrm{O}_{3}\right)$ and three peel angles of $90^{\circ}, 135^{\circ}$ and $180^{\circ}$, peel experiments had been carried out [24].

In order to quantify the separation displacement, we fitted the interfacial separation shapes for the $\mathrm{Al}$ film/ $\mathrm{Al}_{2} \mathrm{O}_{3}$ substrate system. Fig. 2 shows four typical cases. There is a fracture process zone at the crack tip of the interface fracture between the metal film and the ceramic substrate. It can be seen from the experimental figures that the film after peel test has a smooth curve shape in the fracture process zone. We selected the x-axis origin at the maximum separation displacement, as seen in Fig. 2(a). By fitting the interface shape of the metal films after peel test, we found that the relationship between the separation displacement $\delta$ and the ratio of the $\mathrm{x}$-axis to the size of the fracture process zone $x / L$ is of exponential form (the fitting parameters are shown in Fig. 2). Therefore, 

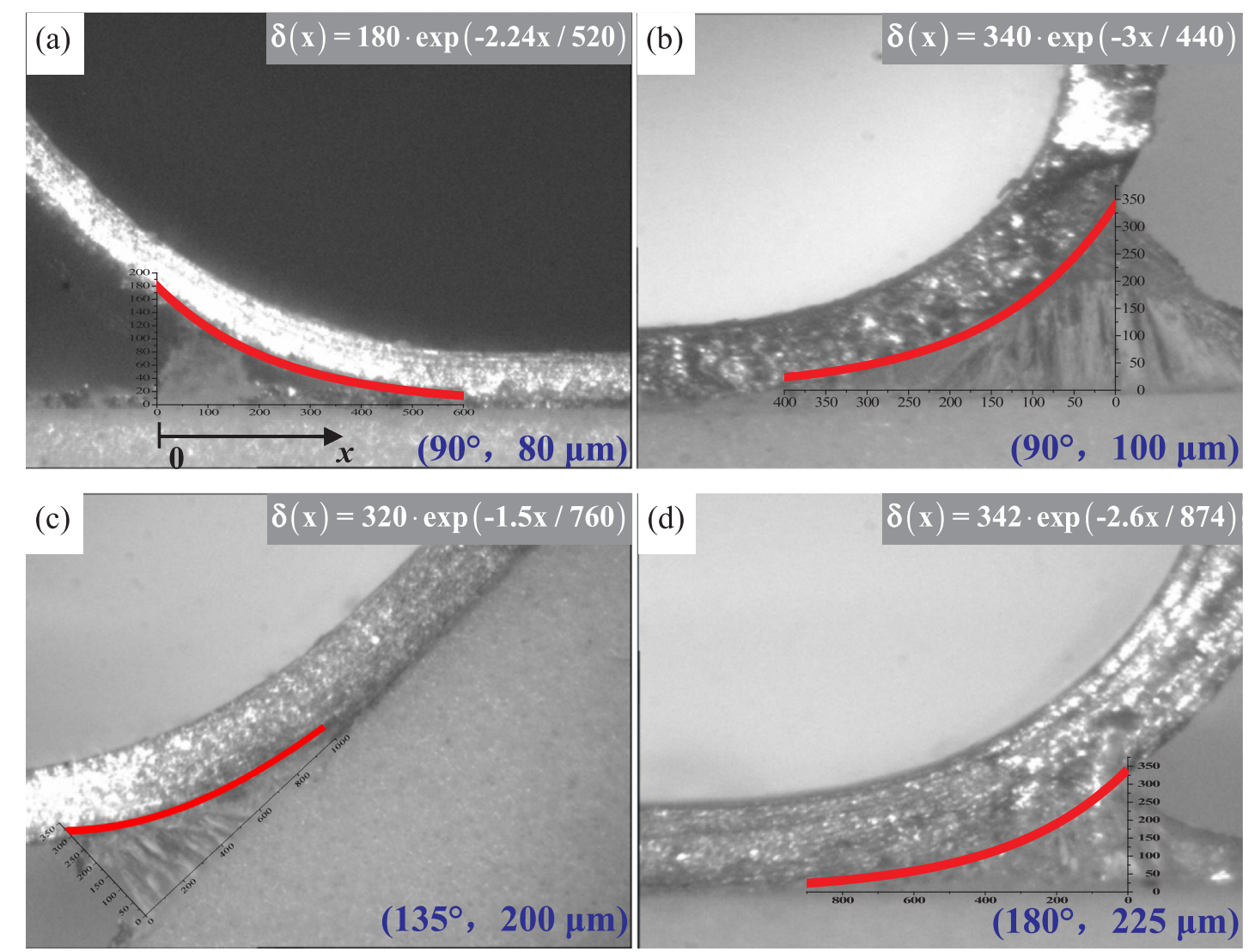

Fig. 2. Fitting curves of interfacial separation shapes of the peel test for the $\mathrm{Al}$ film/ $/ \mathrm{Al}_{2} \mathrm{O}_{3}$ substrate system. The upper right corner shows the fitting function of the separation displacement, and the lower right corner shows the peel angle and film thickness $(\Phi, h)$. The experimental SEM images are taken from Ref. [24].

we assume the form of the interfacial separation displacement as follows

$$
\delta(x)=\delta_{f} \cdot \exp (-k x / L)
$$

where $\delta_{f}$ is the maximum separation displacement, $L$ is the length of the fracture process zone, $k$ is a constant called deformation concentration coefficient, which represents the influence of interfacial separation shape of the peel test to the interfacial separation strength.

Substituting Eq. (20) into Eq. (13), the normalized interfacial separation stress considering the interface effect can be expressed as the function of the following parameters

$$
\frac{t_{m}}{\sigma_{Y}}=f\left(k, \frac{x}{L}, \frac{\delta_{f}}{L}, \frac{\hat{\sigma}}{\sigma_{Y}}, \frac{\Gamma}{\sigma_{Y} \cdot L}, \frac{\gamma}{\sigma_{Y} \cdot L}\right)
$$

where $\sigma_{Y}$ is the traditional yield strength. $(\hat{\sigma}, \Gamma)$ are the interfacial parameters in the cohesive zone model. For general metals, we choose $\sigma_{Y}=10^{2} \mathrm{MPa}, \gamma=1 \mathrm{~J} / \mathrm{m}^{2}$. It can be seen that when the size of the fracture process zone at the macro-/micro scale, the range of the parameter $\gamma /\left(\sigma_{Y} \cdot L\right)$ is on the order of $10^{-3} \sim 10^{-2}$, while if the size of the fracture process zone at the nano-/atomic scale, the range of $\gamma /\left(\sigma_{Y} \cdot L\right)$ is on the order of $10^{-1} \sim 10$. Therefore, here we take the parameter $\gamma /\left(\sigma_{Y} \cdot L\right)$ as the indicator to measure the scale of the fracture process zone.

Fig. 3 shows the interfacial separation stress when the size of the fracture process zone at micrometer scale. Taking the traditional interfacial strength $\hat{\sigma}$ from 1 to 10 times the yield stress $\sigma_{Y}$, the microscale interfacial strengths are all equal to the traditional interfacial strengths. There is no obvious enhancement, which indicates that the influence of the interface effect can be negligible when the fracture process zone is at the micrometer scale.

The trans-scale interfacial separation strength is shown in Fig. 4(a). When the parameter $\gamma /\left(\sigma_{Y} \cdot L\right)$ takes the values in the range of $10^{-3} \sim 10^{-2}$, the size of the fracture process zone is at the macro-/micro scale, the peak of the normalized parameter $t_{m} / \sigma_{Y}$, i.e. the interfacial separation strength, satisfies $t_{m} / \sigma_{Y}=\hat{\sigma} / \sigma_{Y}$, which is the traditional interfacial strength. That is to say, when the scale of the fracture process zone is relatively large, the influence of the interface effect on the interfacial separation strength is negligible. However, when the size of the fracture process zone at the nano-/atomic scale $\left(\gamma /\left(\sigma_{Y} \cdot L\right)\right.$ takes the values on the order of $\left.10^{-1} \sim 10\right)$, the interfacial separation strength is significantly enhanced, even can achieve more than 10-18 times $\sigma_{Y}$. In this case, the interface 


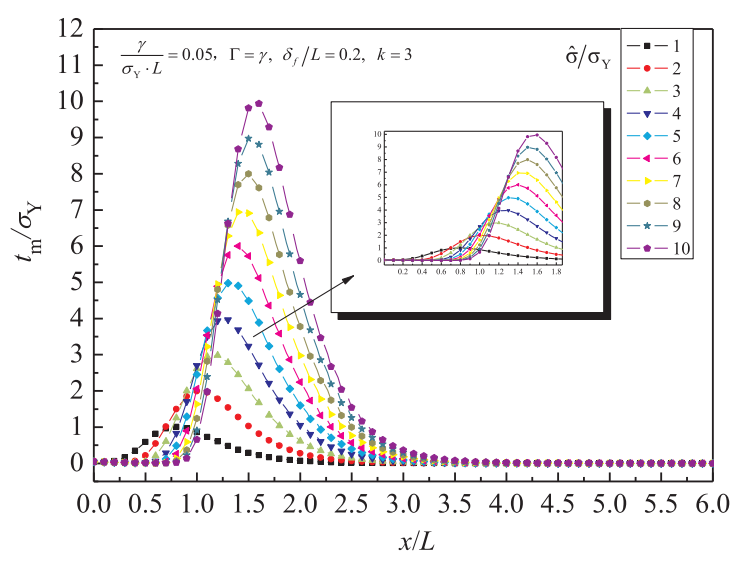

Fig. 3. $\mu \mathrm{m}$-scale interfacial separation strength.
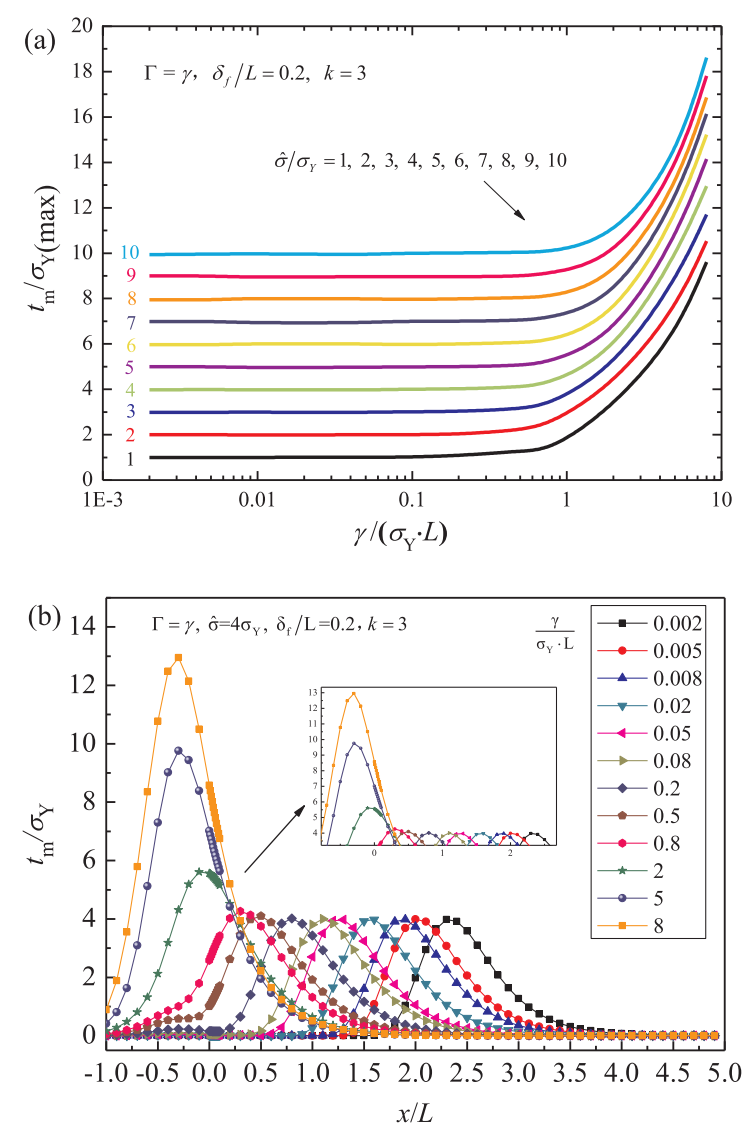

Fig. 4. Trans-scale interfacial separation strength.

effect plays an important role and cannot be neglected. This effect is especially significant when the parameter $\gamma /\left(\sigma_{Y} \cdot L\right)$ is greater than 1. Take the case of $\hat{\sigma} / \sigma_{Y}=4$ for example, the relationship between interfacial separation stress and the position of the peeling thin film is shown in Fig. 4(b). As the value of parameter $\gamma /\left(\sigma_{Y} \cdot L\right)$ increases, the position where the interfacial separation stress is maximum becomes closer to the maximum separation displacement.

Fig. 5 shows the interfacial separation strength corresponding to different scales of fracture process zones and different values of $k$. The constant $k$ is called deformation concentration coefficient, which represents the influence of interfacial separation shape of the peel test to the interfacial separation strength. It can be seen that the interfacial separation strength increases with the increase of the values of $k$, and the magnitude of its increase is enhanced as the parameter $\gamma /\left(\sigma_{Y} \cdot L\right)$ increases. That is to say, the influence of the interfacial separation shape to interfacial separation strength is enhanced when the size of the fracture process zone from macroscopic scale to nano-/micrometer scale. When the value of $k$ is relatively large, the size effect of the interfacial separation strength 


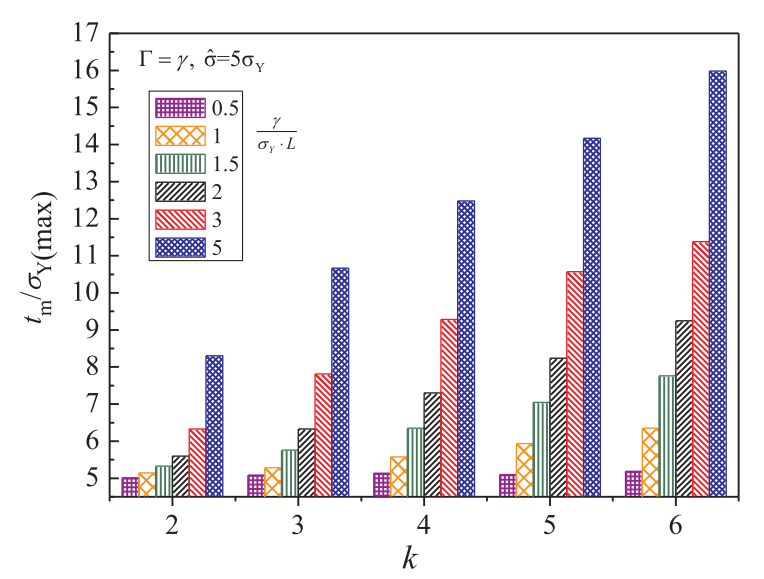

Fig. 5. Influence of deformation concentration coefficient to interfacial separation strength.

becomes more significant.

It is worth noting that from strict derivation based on the energy balance (Eqs. (3), (4) and (11)) and from above application to the peel test of thin film delamination, we have found that the curvature change of interfacial discontinuity lines has a considerable influence on the interface separation strength. This phenomenon is mainly caused by the interaction of interfacial residue energy with the interface bending deformation. This phenomenon is very similar to the stiffened phenomenon happened for a fixed-fixed nanowire under bending [35].

\section{Interfacial energy release rate of peel test considering strain gradient effect}

In this section, the interfacial energy release rate of peel test will be analyzed based on the strain gradient theory and the experimental results. Meanwhile, the material length scale parameters in the strain gradient theory are determined by comparing the measurement results of the peel test with the prediction results of the strain gradient theory.

In terms of theoretical prediction, the strain gradient theory used here is the Wei-Hutchinson strain gradient plasticity theory $[27,29]$. For the problem of the peel test for the metal film along the ceramic substrate, the film can be treated as a strain gradient plasticity material, and the ceramic substrate is an elastic material. In addition, the interface cohesive model is considered. The normalized interfacial energy release rate of the peeling process can be expressed by the following parameter relation as

$$
\frac{G}{\Gamma}=f\left(\frac{E}{\sigma_{Y}}, v, N, \frac{\hat{\sigma}}{\sigma_{Y}}, \frac{l}{R_{0}}\right)
$$

where $E, \nu, N$ are the Young's modulus, Poisson's ratio and the power hardening index of the metal thin film, respectively. $l$ is the material length scale parameter in the strain gradient theory, $R_{0}$ is the size of active plastic zone in the small scale yielding case, which can be expressed as

$$
R_{0}=\frac{E \Gamma}{3 \pi\left(1-\nu^{2}\right) \sigma_{Y}^{2}}
$$

Its value is about 1-5 $\mu \mathrm{m}$ for the metal thin film/ceramic substrate system.

The relations between normalized energy release rate $G / \Gamma$ with the peak separation stress $\hat{\sigma} / \sigma_{Y}$ for different values of $l / R_{0}$ were obtained by the strain gradient theory simulation [32], in which the parameters were taken as $E / \sigma_{Y}=300, \nu=0.3, N=0.1$, as shown in Fig. 6.

A lot of experimental evidence for the metal thin film/ceramic substrate bonded system indicates that the yielding stress of the peeling thin film is inversely proportional to the thin film thickness $[37,38]$. The variation in yielding stress $\sigma_{Y}$ of aluminum and copper thin films with the film thickness $h$ are shown in Fig. 7. In the figure the simulations of the experimental data are also shown by straight lines, which are approximately related with the thin film thickness

$$
\sigma_{Y}=\sigma_{Y}^{0}\left[1+\sqrt{h_{0} / h}\right]
$$

where the simulation parameters $\left(\sigma_{Y}^{0}, h_{0}\right)$ are given in Fig. 7.

Substituting Eq. (24) into Eq. (22) gives

$$
\frac{G}{\Gamma}=f\left(\frac{E}{\sigma_{Y}}, \nu, N, \frac{\hat{\sigma}}{\sigma_{Y}^{0}\left[1+\sqrt{h_{0} / h}\right]}, \frac{l}{R_{0}}\right)
$$

Combining with the strain gradient theoretical results in Fig. 6 and Eq. (25), we can get the theoretical prediction relationship between the normalized interfacial energy release rate $G / \Gamma$ and the film thickness $h$, as the solid lines shown in Fig. 9. 


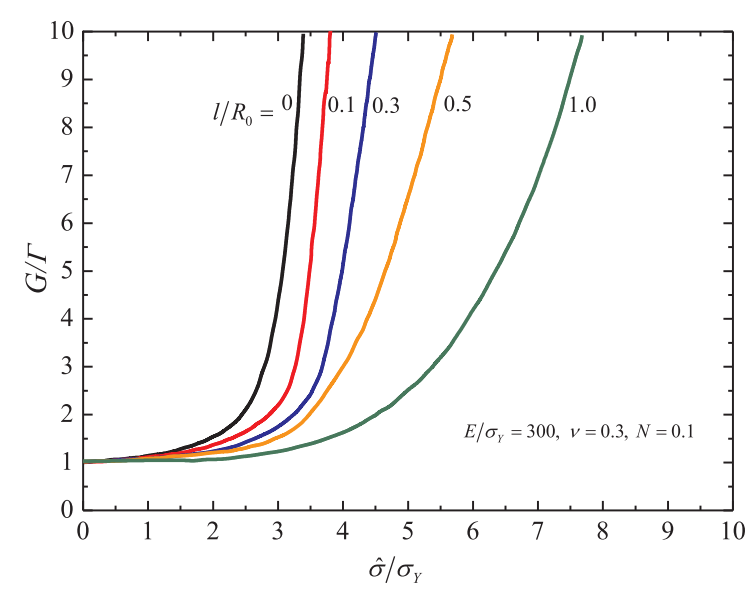

Fig. 6. Parameter relations between interfacial energy release rate with peak separation stress [32].

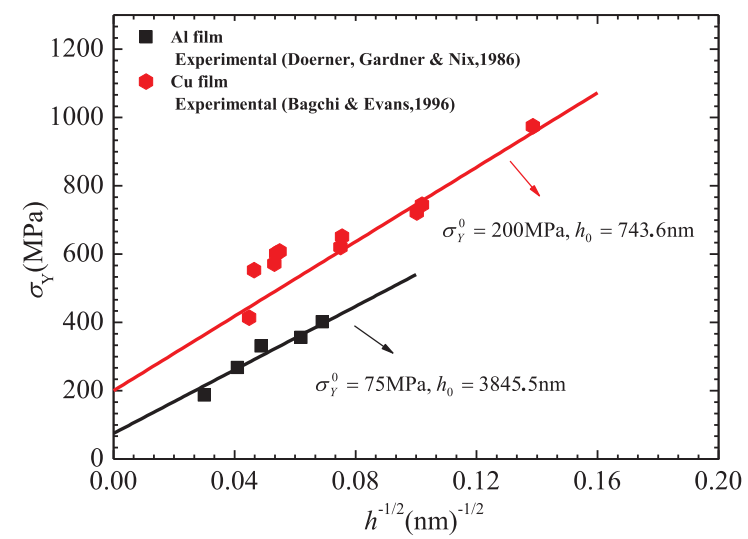

Fig. 7. Variations of yielding stress vs. thin film thickness. Scatter points represent experimental results [37,38] and the solid lines represent simulation results.

In terms of experimental measurements, from the peel test results [24], the relationships between the peel force $P$ and the film thickness $h$ have been given, as shown in Fig. 8. The interfacial energy release rate in the peel test can be expressed as follows

$$
G=P(1-\cos \Phi)
$$

where $P$ is the steady-state peel force, $\Phi$ is peeling angle. Combining with the relationship between $P$ and $h$, we can get the normalized interfacial energy release rate $G / \Gamma$ in different thin film thickness $h$, as the scatter points shown in Fig. 9.

By comparing the prediction results of the strain gradient theory with the experiment results of the relationship between $G / \Gamma$ and

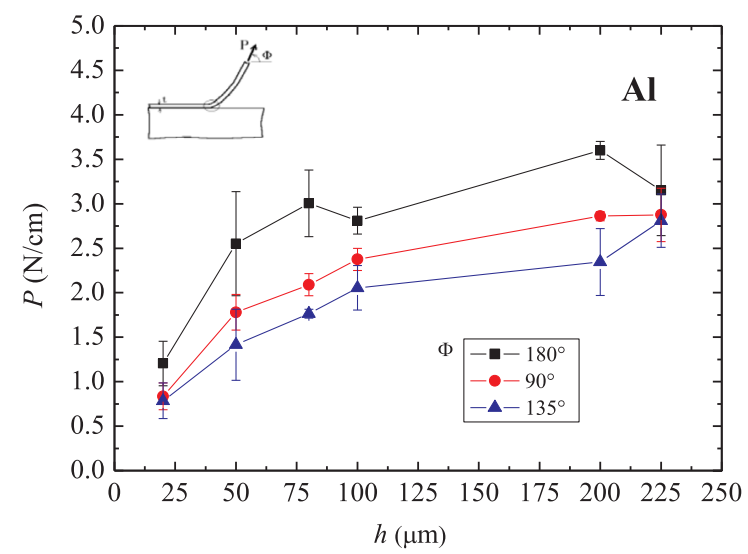

Fig. 8. Experimental results in Ref. [24]: variations of peel force vs. thin film thickness for three peel angles. 

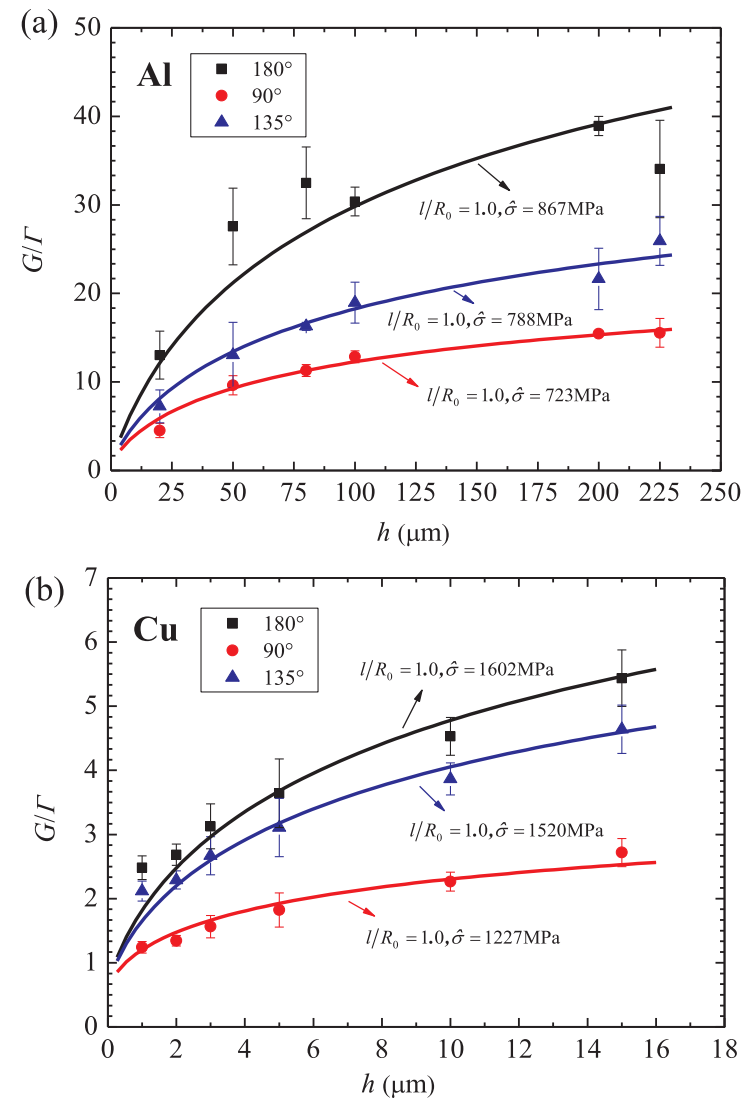

Fig. 9. Relations between interfacial energy release rate with thin film thickness. Comparison of experimental results (scatter points) with theoretical prediction results (solid lines).

$h$, the material length scale parameters of $\mathrm{Al}$ and $\mathrm{Cu}$ are obtained, as shown in Fig. 9. For the peel tests of the two metals at different peeling angels, the theoretical predictions are in good agreement with the experimental measurements when $l=R_{0}$. That is to say, the material length scale parameters could be regarded as the size of active plastic zone in the small scale yielding case. According to Eqs. (23) and (24), we can get the values of the material length scale of $\mathrm{Al}$ and $\mathrm{Cu}$ metals are $4.7 \mu \mathrm{m}$ and $4.27 \mu \mathrm{m}$, respectively, which is consistent with the results obtained by others through experiments [30,31].

\section{Trans-scale interface fracture characterization for peel test}

In our previous work, it was found that the effectiveness of the analytical models used for the peel test were different for the interfaces with different interfacial separation strength. The stronger interface corresponds to the nano-/micro-scale fracture process zone size $L p$, and the weaker interface corresponds to the longer fracture process zone, such as the millimeter scale. One can use the bending model to describe a macroscopic peeling process, and use the conventional 2D elastoplastic theory to describe a small scale peeling process, additionally, use the strain gradient plasticity model to describe a microscale peeling process [24]. From the present researches, we find that a nanoscale peeling process should be described based on the TSM theory models. Summarizing the effectiveness of above several analytical models can be sketched in Fig. 10. It can be seen that there are size effects on the interfacial separation strength of the metal film peel test, and the trans-scale behaviors can be described by different theories which correspond to different fracture process zone sizes. That is to say, the fracture process zone can be considered as an important factor to characterize the trans-scale interface fracture behaviors.

\section{Conclusion}

In the present research, the trans-scale interface fracture behaviors of peel test for the metal film/ceramic substrate systems are investigated and characterized. Both cases considering interface effect and strain gradient effect are studied, respectively. Interface effect dominated zone is implemented. In addition, the material length scale parameters are determined through combining the peel experimental results with numerical simulation based on the trans-scale mechanics theory, the cohesive zone model as well as the virtual internal bond model.

The present results show that when the fracture process zone size is at the nanometer scale or even smaller scale, the interfacial 


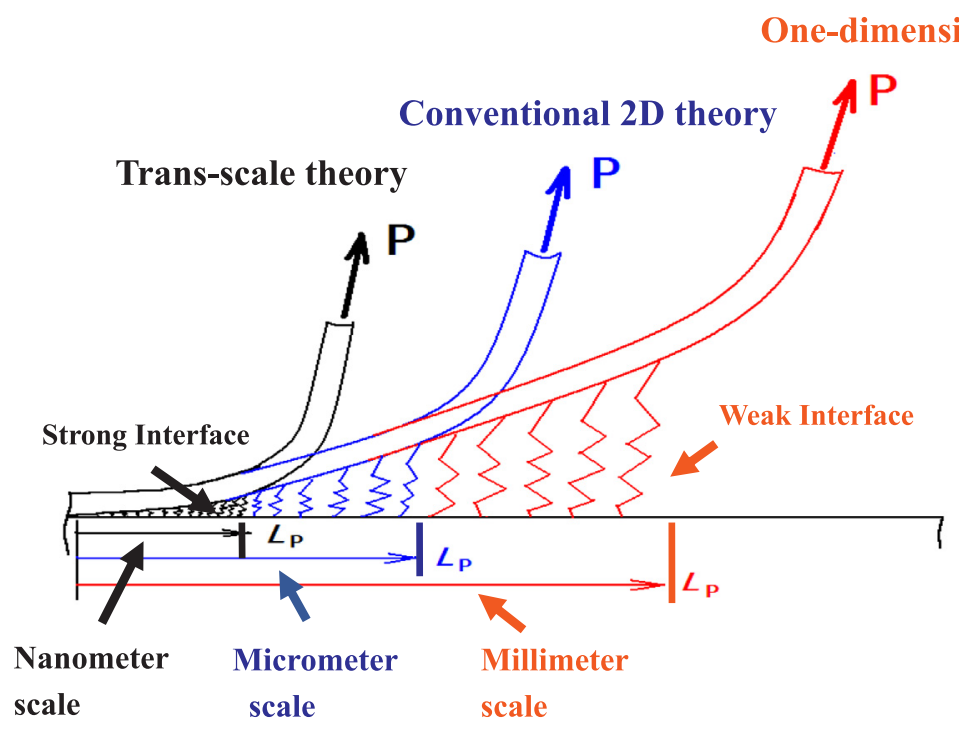

Fig. 10. Interface fracture characteristics of peel tests correspond to different fracture process zone sizes. One dimensional theory (bending theory) is suitable for describing a weak bonded interface case; Conventional two-dimensional theory is suitable for describing a medium strength interface; Trans-scale mechanics theory can be used to describe a strong bonded interface case.

separation strength can reach more than ten times the yield stress, which is much higher than the conventional interfacial cohesive strength. Then, the interfacial energy release rate is characterized by the strain gradient theory. Through comparing the theoretical prediction results of peel test with the experimental results, the material length scales of aluminum and copper are obtained. For the peel test of the metal film/ceramic substrate systems, the material length scale parameters could be connected with the size of the active plastic zone in the small scale yielding case.

Different analytical models used for the peel test correspond to different interfacial separation strength and fracture process zone sizes. The fracture process zone can be considered as an important factor to characterize the trans-scale interface fracture behaviors in peel test.

\section{Declaration of Competing Interest}

The authors declared that they have no conflicts of interest to this work.

We declare that we do not have any commercial or associative interest that represents a conflict of interest in connection with the work submitted.

\section{Acknowledgements}

This work is supported by the National Natural Science Foundation of China (No. 11972347, 11502273, 11432014, 11672301, 11890681 and 11521202) and the Strategic Priority Research Program of the Chinese Academy of Sciences (Grant No. XDB22040501).

\section{Appendix A. The cohesive zone model and the virtual internal bond model}

The cohesive zone model (CZM) [26,33] assumes that there is a fracture process zone near the crack tip, which is characterized by a traction-separation law between the separation displacement $\delta$ and the cohesive stress $\sigma$. Generally, the peak value of the traction and the area under the traction-separation curves are two key parameters, which are denoted as the interfacial strength $\hat{\sigma}$ and the interfacial toughness $\Gamma$, respectively. The cohesive zone model presents a fracture criterion. It is generally considered that the interface crack propagates when the stress state at crack tip satisfies that the maximum interfacial cohesive stress reaches to $\hat{\sigma}$ and the interfacial cohesive energy arrives to $\Gamma$.

The virtual internal bond model (VIB) [39,40] considers a solid with many randomly distributed material particles connected by internal cohesive bonds, in which a cohesive-type law is incorporated directly into the constitutive model. This is achieved by incorporating a randomized network of cohesive bonds into the constitutive law of the material via the Cauchy-Born rule. The VIB model allows one to embed the fracture criterion into the constitutive model so that the physical process of crack initiation and growth becomes a coherent part of the simulation. There is no longer a need for a separate fracture criterion of crack extension. This is also the difference between VIB and CZM when describing the interface fracture behaviors.

The two parameter phenomenological cohesive force law adopted in the VIB model can be expressed as 


$$
U^{\prime}\left(l_{b}\right)=A\left(l_{b}-l_{b 0}\right) \exp \left(-\frac{l_{b}-l_{b 0}}{B}\right)
$$

where $U(l)$ denotes the potential energy function which describes the cohesive bonds; $l_{b 0}, l_{b}$ indicate the length of the bond before and after the deformation; the constants $A$ and $B$ represent the initial modulus and the corresponding change of the bond length at the maximum cohesive strength, respectively. We assume that $\delta=l_{b}-l_{b 0}$, the interfacial cohesive stress can be obtained from Eq. (A.1),

$$
\sigma=A \cdot \delta \cdot \exp \left(-\frac{\delta}{B}\right)
$$

when $\delta=B$, the interfacial cohesive stress is maximum, and can be written as

$$
\sigma_{\max }=A B \cdot \mathrm{e}^{-1}
$$

and the interfacial cohesive energy is given by

$$
\int_{0}^{\infty} \sigma d \delta=\int_{0}^{\infty} A \cdot \delta \cdot \exp \left(-\frac{\delta}{B}\right) d \delta=A B^{2}
$$

Combining with CZM, we have

$$
\left\{\begin{array}{c}
\sigma_{\max }=\hat{\sigma} \\
\int_{0}^{\infty} \sigma d \delta=\Gamma
\end{array}\right.
$$

The constants $A$ and $B$ in Eq. (A.1) can be obtained

$$
A=\frac{\hat{\sigma}^{2} \cdot \mathrm{e}^{2}}{\Gamma}, B=\frac{\Gamma}{\hat{\sigma} \cdot \mathrm{e}}
$$

Substituting them into Eq. (A.2), the interfacial cohesive stress based on the conventional interface models can be expressed as

$$
\sigma=\left(\frac{\hat{\sigma}^{2} \cdot e^{2}}{\Gamma}\right) \cdot \delta \cdot \exp \left(-\frac{\delta \cdot \hat{\sigma} \cdot e}{\Gamma}\right)
$$

\section{References}

[1] Barda H, Rabkin E. Improving the thermal stability of nickel thin films on sapphire by a minor alloying addition of gold. Appl Surf Sci 2019;484:1070-9.

[2] Kumar A, et al. Anomalous diffusion along metal/ceramic interfaces. Nat Commun 2018;9:5251.

[3] Bonu V, Jeevitha M, Kumar VP, Bysakh S, Barshilia HC. Ultra-thin multilayered erosion resistant Ti/TiN coatings with stress absorbing layers. Appl Surf Sci 2019;478:872-81.

[4] Padture NP, Gell M, Jordan EH. Thermal barrier coatings for gas-turbine engine applications. Science 2002;296(5566):280-4.

[5] Bao YP, Chen YF, Lim TT, Wang R, Hu X. A novel metal-organic framework (MOF)-mediated interfacial polymerization for direct deposition of polyamide layer on ceramic substrates for nanofiltration. Adv Mater Interfaces 2019;6(9):1900132.

[6] Tarascon JM, Armand M. Issues and challenges facing rechargeable lithium batteries. Nature 2001;414(6861):359-67.

[7] Wang KS, Zhang FZ, Bordia RK. FEM modeling of in-plane stress distribution in thick brittle coatings/films on ductile substrates subjected to tensile stress to determine interfacial strength. Materials 2018;11(4):497.

[8] Zhang XM, Zhang B, Mu Y, Shao S, Wick CD, Ramachandran BR, et al. Mechanical failure of metal/ceramic interfacial regions under shear loading. Acta Mater 2017; 138:224-36.

[9] Zhuo XR, Beom HG. Interface crack between a thin film and an orthotropic substrate under uniform heat flow. Arch Appl Mech 2016;86:1019-36.

[10] Beom HG, Jang HS, Zhuo XR. Debonding of the interface between a thin film and an orthotropic substrate. Eng Fract Mech 2014;124:217-33.

[11] Fredriksson P, Gudmundson P. Modelling of the interface between a thin film and a substrate within a strain gradient plasticity framework. J Mech Phys Solids 2007;55:939-55.

[12] Shang F, Kitamura T, Hirakata H, Kanno I, Kotera H, Terada K. Experimental and theoretical investigations of delamination at free edge of interface between piezoelectric thin films on a substrate. Int J Solids Struct 2005;42:1729-41.

[13] Tvergaard V, Hutchinson JW. The relation between crack-growth resistance and fracture process parameters in elastic plastic solids. J Mech Phys Solids 1992:40(6):1377-97.

[14] Wei Y, Hutchinson JW. Nonlinear delamination mechanics for thin films. J Mech Phys Solids 1997;45(7):1137-59.

[15] Forschelen PJJ, Suiker ASJ, van der Sluis O. Effect of residual stress on the delamination response of film-substrate systems under bending. Int J Solids Struct 2016;97-98:284-99.

[16] Becker TL, McNancy JM, Cannon RM, et al. Limitations on the use of the mixed mode delaminating beam test specimen: effects of the size of the region of Kdominance. Mech Mater 1997;25(4):291-308.

[17] Kendall K. Thin-film peeling-the elastic term. J Phys D 1975;8(13):1449.

[18] Yin HB, Chen SH, Liang LH, Peng ZL, Wei YG. Quantitative prediction of the whole peeling process of an elastic film on a rigid substrate. J Appl Mech 2018;85(9):091004.

[19] Peng ZL, Wang C, Yang Y, Chen SH. Effect of relative humidity on the peeling behavior of a thin film on a rigid substrate. Phys Rev E 2016;94(3-1):032801.

[20] Chen H, Feng X, Huang Y, Huang YG, Rogers JA. Experiments and viscoelastic analysis of peel test with patterned strips for applications to transfer printing. J Mech Phys Solids 2013;61(8):1737-52.

[21] Kim KS, Aravas N. Elasto-plastic analysis of the peel test. Int J Solids Struct 1988;24(4):417-35.

[22] Wei Y, Hutchinson JW. Interface strength, work of adhesion and plasticity in the peel test. Int J Fract 1998;93(1-4):315-33.

[23] Wei YG. Modeling nonlinear peeling of ductile thin films-critical assessment of analytical bending models using FE simulations. Int J Solids Struct 2004;41(18-19):5087-104.

[24] Wei YG, Zhao HF. Peeling experiments of ductile thin films along ceramic substrates-critical assessment of analytical models. Int J Solids Struct 2008;45(13):3779-92. 
[25] Zhao HP, Wang YC, Li BW, Feng XQ. Improvement of the peeling strength of thin films by a bioinspired hierarchical interface. Int J Appl Mech 2013;15:1350012.

[26] Zhao HF, Wei YG. Determination of interface properties between micron-thick metal film and ceramic substrate using peel test. Int J Fract 2007;144(2):103-12.

[27] Wei Y, Hutchinson JW. Steady-state crack growth and work of fracture for solids characterized by strain gradient plasticity. J Mech Phys Solids 1997;45(8):1253-73.

[28] Fleck NA, Hutchinson JW. Strain gradient plasticity. Hutchinson JW, Wu TY, editors. Advances in Applied Mechanics, Vol. 33. New York: Academic Press; 1997. p. 295-361.

[29] Wei YG. A new finite element method for strain gradient theories and applications to fracture analyses. Eur J Mech A-Solid 2006;25:897-913.

[30] Fleck NA, Muller GM, Ashby MF, Hutchinson JW. Strain gradient plasticity theory and experiment. Acta Metall Mater 1994;42:475-87.

[31] Haque MA, Saif MTA. Strain gradient effect in nanoscale thin films. Acta Mater 2003;51:3053-61.

[32] Wei Y. Microscale mechanics for metal thin film delamination along ceramic substrates. Science in China (Series A) 2000;43(5):509-16.

[33] Wu B, Liang LH, Ma HS, Wei YG. A trans-scale model for size effects and intergranular fracture in nanocrystalline and ultra-fine polycrystalline metals. Comput Mater Sci 2012;57:2-7.

[34] Song JR, Liu JY, Ma HS, Liang LH, Wei YG. Determinations of both length scales and surface elastic parameters for fcc metals. C R Mecanique 2014;342:315-25.

[35] Yao Y, Chen SH. Surface effect in the bending of nanowires. Mech Mater 2016;100:12-21.

[36] Yao Y, Chen SH, Fang DN. An interface energy density-based theory considering the coherent interface effect in nanomaterials. J Mech Phys Solids 2017;99:321-37.

[37] Bagchi A, Evans AG. The mechanics and physics of thin film decohesion and its measurement. Interface Sci 1996;3(3):169-93.

[38] Doerner MF, Gardner DS, Nix WD. Plastic properties of thin films on substrates as measured by submicron indentation hardness and substrate curvature techniques. J Mater Res 1986;1(6):845-51.

[39] Gao HJ, Klein P. Numerical simulation of crack growth in an isotropic solid with randomized internal cohesive bonds. J Mech Phys Solids 1998;46(2):187-218.

[40] Ding JF, Zhang ZN, Yang SQ, Ma ZG, Mou JY. Numerical simulation of thermal-mechanical induced fracture with discretized virtual internal bond. Eng Fract Mech 2018;189:377-89. 